\title{
Prima de riesgo por inflación calculada con el Break-Even Inflation y el modelo dinámico Nelson-Siegel
}

\author{
Inflation Risk Premium Calculated with Break-Even Inflation and the Dynamic Nelson-Siegel Model
}

Prime de risque par inflation calculée avec le Break-Even Inflation et le modèle dynamique Nelson-Siegel

\begin{abstract}
Omar Alexander Ríos
Docente, Departamento de Ciencias Naturales, Pontificia

Universidad Javeriana, Cali - Colombia. Magister en Economía, Pontificia Universidad Javeriana, Bogotá, Colombia. Estadístico profesional, Universidad del Valle.

E-mail: orios@javeriancali.edu.co
\end{abstract}

Luis Eduardo Girón

Docente investigador Grupo GIDR - Categoría D, Departamento de Economía, Pontificia Universidad Javeriana, Cali - Colombia. PhD (c) en Métodos Matemáticos Aplicados a la Economía y las Finanzas, Universidad Nacional de Educación a Distancia, Madrid, España. Maestría en Economía Aplicada, Universidad del Valle, Colombia. Estadístico profesional, Universidad del Valle, Colombia. E-mail: legiron@javerianacali.edu.co

Artículo de investigación científica y tecnológica según clasificación COLCIENCIAS

Recepción: 06/03/2013

Corrección: 28/04/2013

Aprobación: 16/05/2013

\section{Resumen}

En la presente investigación, que se enmarca dentro de la economía y las finanzas, se estima la prima de riesgo por inflación para Colombia a partir de las expectativas de inflación proporcionadas por el indicador Break Even Inflation (BEI). Los modelos utilizados en estudios anteriores de este tipo son estructuralmente complejos en la medida que no son parsimoniosos, pues el número de parámetros a estimar es alto y de difícil comprensión y se concentran en la medición de las expectativas de inflación. Adicional a lo anterior, todos ellos requieren información primaria acerca del comportamiento de las expectativas de inflación a partir de las encuestas realizadas por los bancos centrales. La metodología usada es el filtro Kalman con una representación estado espacio para la ecuación dinámica de Nelson y Siegel. Los datos utilizados son los rendimientos de los títulos nominales y de los títulos indexados a la inflación para el período enero 2003 a septiembre 2010, información pública proporcionada por la Bolsa de Valores de Colombia. Los resultados muestran que la prima de riesgo por inflación para un plazo de cinco años es de 53 puntos básicos.

Palabras clave: prima de riesgo por inflación, expectativas de inflación, break even inflation, modelo estado espacio, filtro de Kalman.

\section{Abstract}

In the present investigation, which is part of the economy and finance, we estimate the inflation risk premium for Colombia from inflation expectations contained in the Break-Even Inflation (BEI) indicator. The models used in previous studies of this type are structurally complex because they are not parsimonious, given that the number of parameters to be estimated is high and difficult to understand, focusing primarily on the measurement of inflation expectations. Additional to this, all require primary information about the behavior of inflation expectations from surveys conducted by the central banks. The methodology used is the Kalman filter with a state space representation for the dynamic Nelson and Siegel equation. The data used are the nominal bond yields and inflation-indexed securities for the period January 2003 to September 2010; public information was provided by the Colombian stock exchange. The results show that the inflation risk premium for a five-year period is 53 basis points.

$$
\text { Keywords: inflation expectations, break-even inflation, inflation risk premium, state space model, Kalman filter. }
$$

\section{Résumée}

Cette recherche, encadrée dans l'économie et les finances, considère la prime de risque par inflation pour la Colombie à partir des expectatives d’inflation proportionnées par l’indicateur Break Even Inflation (BEI). Les modèles utilisés dans des études antérieures de ce type sont structurellement complexes dans la mesure où elles ne sont pas parcimonieuses, car le nombre de 
paramètres à estimer est élevé et difficile à comprendre, et se concentre sur la mesure des expectatives d'inflation. De plus, ils ont tous besoin d'information primaire en relation avec le comportement des expectatives de l'inflation à partir des enquêtes réalisées par les banques centrales. La méthodologie utilisée est le filtre Kalman avec une représentation état-espace pour l'équation dynamique de Nelson et Siegel. Les données utilisées sont les revenus des titres nominaux et des titres indexés à l'inflation pour la période janvier 2003 à septembre 2010, information publique proportionnée par la Bolsa de Valores de Colombia. Les résultats montrent que la prime de risque par inflation pour un délai de cinq ans est de 53 points basiques.

Mots clef: prime de risque par inflation, expectatives d'inflation, break even inflation, modèle état espace, filtre de Kalman.

\section{Introducción}

La teoría neoclásica de la hipótesis de las expectativas racionales, desarrollada inicialmente por Muth (1961), explica cómo los agentes utilizan toda la información disponible para realizar sus predicciones sobre el comportamiento de ciertas variables económicas, planteando además que estas predicciones son eficientes.

La hipótesis se fundamenta en que la economía en general no pierde información y que las expectativas dependen específicamente de la estructura de todo el sistema. Es importante aclarar que en la hipótesis de las expectativas racionales se asumen dos supuestos fundamentales. Primero que los agentes son racionales respecto de la formación de sus expectativas y segundo que los mercados tienden en forma constante al equilibrio general (Lucas, 1972, 1976; Barro et al., 1976).

Ahora bien, la formación de las expectativas por parte de los agentes económicos sobre la inflación, es un indicador importante para los bancos centrales ${ }^{1}$ encargados del diseño de la política monetaria, pues la eficacia de la misma depende de la incertidumbre de estas expectativas y de otros factores.

Ya establecida la importancia de las expectativas de inflación, hay diferentes mecanismos que permiten medirlas en los agentes del mercado. Dos de ellos son las encuestas realizadas periódicamente por los bancos centrales ${ }^{1}$ y los modelos econométricos.

Otro mecanismo que permite establecer la magnitud de las expectativas inflacionarias de los agentes la propone Fisher (1930) con su ecuación, que define la tasa de interés nominal como la suma entre la tasa de interés real y las expectativas de inflación. En su versión más simple esta ecuación se representa de la siguiente manera: $i_{t}^{\text {rom }} \approx i_{t}^{\text {real }}+E_{t}\left[\Pi_{t}+n\right]$

Una de las formas de calcular las expectativas de inflación en los mercados financieros es a través del indicador Break Even Inflation (BEI) que se basa en la ecuación de Fisher, que consiste en la diferencia entre los rendimientos de los bonos nominales y reales para un mismo plazo de vencimiento.

Existen una serie de trabajos orientados a calcular las expectativas de inflación en Colombia y en otras regiones. Ang, Bekaert y Wei (2005), plantean diferentes métodos para el cálculo de las exceptivas de inflación, como modelos ARIMA, regresiones usando medidas de actividad real derivadas de la curva de Phillips, modelos de estructura de términos y medidas basadas en encuestas; no tienen en cuenta la estructura característica de una curva de rendimientos, las cuales son importantes para la simulación de los componentes utilizados en el cálculo de la prima de riesgo. Gaviria y Téllez (2010), mediante el uso de un modelo econométrico y suponiendo racionalidad en las expectativas de los agentes, hallan las expectativas de inflación considerando la dinámica conjunta de las tasas de interés, la inflación y el ciclo de la economía. González, Jalil y Romero (2010) realizan estimaciones de las expectativas de inflación con métodos directos referentes a las encuestas sobre expectativas de inflación y métodos indirectos que se refieren a la obtención de la medida a partir del comportamiento del mercado de deuda pública, con el fin de evaluar la capacidad predictiva de estos métodos con respecto al comportamiento futuro de la inflación, concluyendo que ambos son complementarios.

Las expectativas de inflación calculadas en función del comportamiento de los mercados financieros, a partir de la ecuación establecida por Fisher, ha sido cuestionada por Sack (2000).Teniendo en cuenta que los agentes económicos prefieren evitar o limitar el riesgo en sus inversiones, estos exigirán el pago de una prima de riesgo por inflación cuando sus inversiones se encuentren indexadas con la tasa de interés real ${ }^{2}$. Por tanto, Sack plantea que para obtener una mejor estimación de las expectativas inflacionarias de los agentes se debe restar esta prima de riesgo por inflación del indicador generado por la ecuación de Fisher.

La forma estructural de la prima de riesgo por inflación puede ser obtenida según Adrian y Wu (2009) y Christensen, López y Rudebusch (2010) como la covarianza entre

1. Las estimaciones aquí resultantes no son consistentes con la teoría de las expectativas racionales. Deacon y Derry (2003) sugieren que las encuestas no dan información continua de las expectativas de inflación debido al tiempo que toma recolectar la información. Otra causa es que la información tomada de los agentes puede no ser veraz, debido a que estos no tienen incentivos para contestarla de manera pertinente, tampoco se tiene una ponderación dependiente del mercado en donde opera el agente. Por último, las encuestas solo generan información con un horizonte de proyección en el corto plazo.

2. La inflación tiene un impacto mayor sobre la tasa de interés nominal, ya que reduce el poder adquisitivo de los agentes y disminuye la tasa real de rendimiento sobre cualquier inversión. Es por esto que los inversionistas o agentes racionales, requieren estimar la prima que estarían dispuestos a pagar por minimizar los riesgos asociados con cambios en la inflación. 
la inflación y el factor de descuento estocástico futuro, siendo el factor de descuento estocástico, el método por medio del cual se hallan las rentabilidades de los bonos (nominal y real) a diferentes plazos de rendimiento.

Otros trabajos en los que se desarrolla la descomposición del $\mathrm{BEI}$ en expectativas de inflación y prima de riesgo por inflación son Melo y Granados (2010) que usan un modelo Afín con una representación de estado espacio incluyendo en la estimación las encuestas de expectativas de inflación realizadas por el banco central. Por otro lado, Hördahl y Tristani (2007) estiman la dimensión y dinámica de la prima de riesgo por inflación para la zona Euro; basados en un modelo que infiere de manera conjunta dinámicas macroeconómicas, tomando información acerca de los rendimientos de bonos nominales y bonos indexados al cambio en los precios. De igual forma lo realizan García y Werner (2010) que investigan la transferencia entre los riesgos inflacionarios en las estimaciones macroeconómicas y la prima de riesgo por inflación calculada a partir del funcionamiento de los mercados financieros. En este estudio se examinan algunos hechos estilizados acerca de la estructura de la inflación compensada, las expectativas de inflación y la prima de riesgo inflacionaria en el mercado de bonos de la zona Euro.

Los modelos utilizados en los estudios anteriores son estructuralmente complejos en la medida que no son parsimoniosos, pues el número de parámetros a estimar es alto y de difícil comprensión, concentrándose básicamente en la medición de las expectativas de inflación. Además todos ellos requieren información primaria acerca del comportamiento de las expectativas de inflación a partir de las encuestas realizadas por los bancos centrales.

Con lo anterior, en el presente trabajo se determina la prima de riesgo por inflación utilizando la información contenida en los rendimientos de los bonos nominales y reales a cualquier período de vencimiento. Para ello se aplica el Modelo Dinámico de Nelson y Siegel (DNS) con el fin de descomponer la medición BEI en expectativas de inflación y la prima de riesgo por inflación. La novedad metodológica del modelo desarrollado en la presente investigación radica en tres ejes fundamentales. El primero es que solo utiliza información contenida en los rendimientos de los bonos nominales y reales a cualquier período de vencimiento, que es obtenida de forma libre del funcionamiento de los mercados financieros sin utilizar para el cálculo de la prima las encuestas de las expectativas de inflación. El segundo es la facilidad en la interpretación práctica de los parámetros del modelo, porque estos se basan únicamente en los factores latentes que caracterizan una curva de rendimientos, nivel, pendiente y curvatura. El tercero es que calcula de manera directa el comportamiento de la prima de riesgo por inflación que es un indicador importante para los diferentes agentes del mercado.
Este indicador entendido como la compensación requerida por invertir en títulos indexados a la inflación, es importante para agentes como fondos de pensiones, administradores de los mismos y en general cualquier inversionista informado, porque consigue disminuir de manera eficiente los riesgos por la variación de los precios, teniendo en cuenta el valor o los puntos básicos que deben pagar para indexar sus activos y evitar el riesgo de inflación. Utilizar la prima de riesgo por inflación en las inversiones llevaría a una mejor asignación del precio de los activos financieros con menor incertidumbre.

En el caso de los inversionistas, si estos no se cubren lo suficiente frente a los riesgos de inflación realizarán operaciones tales como vender gran parte sus activos con el fin de minimizar el riesgo inflacionario, con ello deprecian las inversiones generando pérdidas, porque si no se recuperan a un precio menor o igual, el ahorro se pierde. Por otro lado, conociendo el costo de la prima de riesgo por inflación, los inversionistas pueden cubrir sus activos o pasivos ante cambios en la inflación y así conservar el ahorro en términos reales.

Dos ejemplos que ilustran la aplicación e importancia de la prima de riesgo por inflación son lo siguientes:

1. Si un gestor de portafolio, espera que la inflación futura para un plazo de 5 años sea del $4 \%$ medida a través del BEI y quiere obtener una tasa de interés nominal de inversión del 10\% (es decir una tasa de interés real del $6 \%$ ), estaría dispuesto a invertir en títulos indexados a la inflación a una tasa del 5,5\% (a un plazo de 5 años), es decir, que obtendría una tasa nominal del $9,5 \%$ extrayendo una prima de riesgo por inflación de 50 puntos básicos.

2. Cuando el gobierno emite TES UVR que se pueden denotar como títulos de deuda pública indexados a la inflación, el retorno real de estos títulos viene dado por la tasa de interés real y una prima de riesgo por inflación.

\section{Metodología}

El presente trabajo se basa en un marco teórico que está fundamentado en dos teorías. La primera es la teoría económica del riesgo y la incertidumbre de la que se obtiene la derivación teórica de la prima de riesgo por inflación a través de la maximización de la utilidad esperada. La segunda es el modelo estado espacio que es el modelo econométrico del que se obtienen los factores para el cálculo de la prima de riesgo por inflación.

Las etapas seguidas para alcanzar los objetivos propuestos son:

Primero, utilizando la información obtenida del mercado de deuda pública, se calcula el BEI como la diferencia entre los rendimientos de los títulos nominales y reales para diferentes plazos de vencimiento. Segundo, 
siguiendo el modelo de Nelson y Siegel (1987) en una estructura estado espacio, se obtienen de los rendimientos de los bonos nominales y reales, los factores latentes de la curva de rendimientos, nivel, pendiente y curvatura. Tercero, se procede a calcular las tasas de interés libre de riesgo y el factor de descuento estocástico en función de los factores latentes. Finalmente, se procede al cálculo de la prima de riesgo por inflación, que corresponde a la covarianza entre el factor de descuento estocástico en bonos reales y el cambio de precios futuros, que es una función de las tasas de interés libre de riesgo para los bonos nominales y reales. A partir de este cálculo se obtienen las expectativas de inflación del indicador BEl.

\subsection{La ecuación de Fisher y el BEI}

Los bancos centrales calculan el BEI como la diferencia entre las tasas de interés de los rendimientos de los bonos nominales y reales ${ }^{3} i-r$ con el propósito de aproximarse a las expectativas de inflación del mercado. Se puede observar en la ecuación de Fisher, que si la prima de riesgo por inflación se asume constante, el BEl se diferencia de las expectativas de inflación por esa constante. Por tanto, cambios en el BEI estiman de manera precisa los cambios en las expectativas de inflación, incrementos en la prima de riesgo inflacionaria produce incrementos en la tasa BEl.

El BEl se basa en la ecuación de Fisher (1930):

$i_{t}^{\text {nom }} \approx i_{t}^{\text {real }}+E_{t}\left[\pi_{t}+n\right]$

Donde

$i_{t}^{\text {nom }}$ corresponde a la tasa de interés nominal

$i_{t}^{\text {real }}$ es la tasa de interés real

$E_{t}\left[\pi_{t}+n\right]$ representa las expectativas de inflación, que asimismo puede ser representada por la diferencia entre los rendimientos de un bono nominal y uno real al mismo plazo de madurez para un vencimiento de plazo $\mathrm{t}$ :

$B E I_{t}=y_{t}^{\text {nom }, \tau}-y^{\text {real }, \tau} \approx E_{t}\left[\pi_{t+\tau}\right]$

Naturalmente si los agentes demandan retornos similares para ambas clases de bonos tanto nominales como reales, entonces la medición del BEI como se ha expresado antes representa de manera idónea la estimación de la inflación. Sack (2000), identifica que las razones más importantes por las cuales los retornos esperados de los bonos nominales y reales difieren en medida son: 1) La prima de inflación asociada con el retorno nominal. 2) Diferencias en la liquidez de los bonos nominales e indexados, los volúmenes transaccionales a favor de los bonos nominales es mayor. 3) El impacto de la senda de las tasas de interés reales es diferente para ambos bonos (el valor real de los bonos nominales pierde fuerza por influencia de la inflación y con esto su duración se hace más corta). 4) Por lo anterior existen diferencias en el riesgo de tasa de interés real que enfrentan los bonos por sus diferentes períodos de madurez.

Es así que Shen (2006), define que el rendimiento de un título nominal tiene tres componentes: el rendimiento real, la tasa de inflación promedio esperada durante la vida del título y la prima de riesgo de la inflación (que compensa a los inversionistas por el riesgo que la tasa de inflación sobre la vida del título pueda ser más alta que la esperada).

Debido a que la inflación erosiona el poder adquisitivo de los pagos del nominal, el rendimiento correspondiente para los inversionistas no es del nominal sino el real.

Se sabe que la inflación tiene un comportamiento completamente aleatorio en la realidad, porque depende de condiciones no controlables del mercado, la tasa nominal de rendimiento incluye la tasa real, la inflación esperada, una prima de riesgo de inflación e implícitamente una prima de riesgo por liquidez. Dependiendo de la credibilidad que tengan los inversionistas en las autoridades monetarias respecto a sus decisiones, cambiará el valor de la prima de riesgo por inflación.

\subsection{Factor de descuento estocástico}

Es necesario antes de definir la prima de riesgo por inflación describir el factor de descuento estocástico el cual trae a valor presente el precio de los bonos a diferentes plazos de vencimiento.

El factor de descuento estocástico es el resultado que resuelve el problema de maximización de la utilidad para un inversionista representativo. En este modelo se puede tranzar un activo en diferentes períodos y se maximiza las expectativas de una función de utilidad separable descrita de la siguiente forma:

$\operatorname{Max} E_{t}\left[\sum_{j=o}^{\infty} \delta^{j} U\left(C_{t+j}\right)\right.$

Donde:

$\delta$, es el factor de descuento temporal

$U\left(C_{t+j}\right)$ es la utilidad del consumo para el inversionista en el período $t+j$.

Después de algunos cálculos la solución al proceso de maximización genera la siguiente expresión:

$$
M_{t}^{\text {real }}=\frac{M \delta U^{\prime}\left(C_{t+1}\right)}{U^{\prime}\left(C_{t}\right)}
$$

3. El rendimiento real de un TES indexado a la UVR también se puede denotar como un título de deuda pública indexado a la inflación, donde el retorno real de estos títulos viene dado por la tasa de interés real y una prima por inflación. La prima de riesgo de inflación es la compensación requerida por los inversionistas por invertir en títulos indexados a la inflación, riesgo al que no se ven enfrentados los títulos convencionales del gobierno. 
Donde

$\mathrm{M}_{t+1}^{\text {real }}$ representa el factor de descuento estocástico real o real pricing Kernel, que es equivalente a la tasa marginal de sustitución intertemporal del inversionista que compra el bono. Lo que implica que el comportamiento del factor de descuento intertemporal es tal, que si la utilidad marginal es decreciente, se espera que cuando el consumo agregado y la inflación sean altos, el factor de descuento tiende a ser pequeño.

\subsection{Modelo dinámico de Nelson y Siegel (DNS) y modelo estado espacio}

Diebold y Li (2002) muestran una representación de la ecuación de Nelson y Siegel (1987), donde se ve que los parámetros del modelo $\beta_{o}, \beta_{1}, \beta_{2}$ de Nelson y Siegel, pueden ser interpretados como una representación dinámica de tres factores latentes variantes en el tiempo conocidos como nivel, pendiente y curvatura (N, P, C, por sus iniciales) respectivamente, entonces el modelo de Nelson y Siegel puede ser escrito de la siguiente manera:

$$
y(\tau)=N_{t}+P_{t}\left(\frac{1-e^{-\lambda \tau}}{\lambda \tau}\right)+C_{t}\left(\frac{1-e^{-\lambda \tau}}{\lambda \tau}-e^{-\lambda \tau}\right) 4
$$

El presente trabajo se centra en la versión más simple de un modelo DNS, que asume independencia entre los factores que integran el modelo de Nelson-Siegel. Diebold y Li (2006), representan las variables de estado como una dinámica de los movimientos del nivel $\left(\mathrm{N}_{\mathrm{t}}\right)$, la pendiente $\left(P_{t}\right)$ y la curvatura $\left(C_{t}\right)$, en un vector autorregresivo de primer orden VAR(1), así el sistema de estado espacio estará determinado por la ecuación de transición:

$$
\left(\begin{array}{l}
N_{t}-\mu_{L} \\
P_{t}-\mu_{s} \\
C_{t}-\mu_{C}
\end{array}\right)=\left(\begin{array}{ccc}
a_{11} & 0 & 0 \\
0 & a_{22} & 0 \\
0 & 0 & a_{33}
\end{array}\right)\left(\begin{array}{l}
N_{t-1}-\mu_{N} \\
P_{t-1}-\mu_{P} \\
C_{t-1}-\mu_{C}
\end{array}\right)+\left(\begin{array}{l}
n_{t}(N) \\
n_{t}(P) \\
n_{t}(C)
\end{array}\right) t=1,2, \ldots T
$$

Los choques estocásticos $n_{t}(N), n_{t}(P)$ y $n_{t}(C)$ tienen matriz de covarianzas:

$$
\left(\begin{array}{lll}
q_{11}^{2} & 0 & 0 \\
0 & q_{22}^{2} & 0 \\
0 & 0 & q_{33}^{2}
\end{array}\right)
$$

La ecuación de medición que relaciona los rendimientos:

$$
\left(\begin{array}{c}
y_{t}\left(\tau_{1}\right) \\
y_{t}\left(\tau_{2}\right) \\
\vdots \\
y_{t}\left(\tau_{N}\right)
\end{array}\right)=\left(\begin{array}{cc}
1 \frac{1-e^{-\lambda \tau_{1}}}{\lambda \tau_{1}} \frac{1-e^{-\lambda \tau_{1}}}{\lambda \tau_{1}}-e^{-\lambda \tau_{1}} \\
1 \frac{1-e^{-\lambda \tau_{2}}}{\lambda \tau_{2}} \frac{1-e^{-\lambda \tau_{2}}}{\lambda \tau_{2}}-e^{-\lambda \tau_{2}} \\
\vdots \\
1 \frac{1-e^{-\lambda \tau_{N}}}{\lambda \tau_{N}} \frac{1-e^{-\lambda \tau_{N}}}{\lambda \tau_{N}}-e^{-\lambda \tau_{N}}
\end{array}\right)\left(\begin{array}{c}
N_{t} \\
P_{t} \\
C_{t}
\end{array}\right)+\left(\begin{array}{c}
\varepsilon_{t}\left(\tau_{1}\right) \\
\varepsilon_{t}\left(\tau_{2}\right) \\
\vdots \\
\varepsilon_{t}^{\vdots}\left(\tau_{N}\right)
\end{array}\right)
$$

Así, el sistema de estado espacio puede ser escrito de la siguiente manera:

$X_{t}=(I-A) \mu+A X_{t-1}+n_{t}$

La ecuación de medición queda expresada como: $y_{t}=B X_{t}+\varepsilon_{t}$

Con la estructura de error:

$\left(\begin{array}{l}n_{t} \\ \varepsilon_{t}\end{array}\right) \sim W N\left[\left(\begin{array}{l}o \\ o\end{array}\right),\left(\begin{array}{ll}Q & o \\ o & H\end{array}\right)\right]$

El vector de estado bajo un modelo DNS, viene dado por los factores NPC, denotado como: $X_{t}^{i}=\left(N_{t}^{i}, P_{t}^{i}, C_{t}^{i}\right)$ Christensen, Diebold y Rudebusch (2007), demuestran que la tasa libre de riesgo para bonos nominales y reales, se toma como la suma de los factores $\mathrm{N}$ y $\mathrm{P}$ :

$r_{t}^{i}=N_{t}^{i}+P_{t}^{i} i=N, R$

El algoritmo de Kalman, resuelve el sistema dinámico optimizando por mínimos cuadrados lineales. Los errores tanto en la ecuación de estado como en la ecuación de medición deben tener una distribución ruido blanco y deben ser ortogonales al sistema ${ }^{5}$ :

$\left(\begin{array}{l}\mathrm{n}_{\mathrm{t}} \\ \varepsilon_{\mathrm{t}}\end{array}\right) \sim W N\left[\left(\begin{array}{l}0 \\ o\end{array}\right),\left(\begin{array}{ll}Q & 0 \\ O & H\end{array}\right)\right]$

$E\left(A_{o} n_{t}^{\prime}\right)=o$

$E\left(A_{o} \varepsilon_{t}^{\prime}\right)=0$

Para la caracterización del modelo se tendrán en cuenta las tasas mensuales de negociación de los TES (títulos de deuda pública doméstica con rendimiento fijo) denominados en tasa fija y UVR (títulos de deuda pública con tasa de rendimiento variable atados a la inflación), realizadas en los diferentes sistemas transaccionales tanto del Banco de la República como de la Bolsa de Valores de Colombia con cierres diarios. Para títulos nominales se toman en cuenta, períodos de madurez de $3,12,24,36$ y 60 meses y en títulos reales, períodos de madurez a 24,36 y 60 meses.

Estas series fueron ajustadas con el fin de obtener una curva continua de rendimientos de ambos activos para que coincidan en los plazos y obtener una medida más ajustada del BEl.

Los TES son denominados dentro de Colombia como un activo con el menor riesgo de crédito porque son emitidos por la nación con el fin de cumplir con las necesidades de presupuesto, también los utiliza el Banco Central para realizar su política monetaria.

4. Christensen, Diebold y Rudebusch (2010), realizan la prueba de la solución del sistema.

5. Para los análisis que siguen a continuación se asumirá que tanto las matrices Q y H son diagonales, lo cual implica en el caso de la matriz $\mathrm{H}$, que las desviaciones de los rendimientos de los títulos para distintos períodos de madurez no se encuentran correlacionados, y en el caso de la matriz $Q$ se asume que los choques de los tres factores latentes tampoco se encuentran correlacionados. 


\subsection{Prima de riesgo por inflación}

El factor de descuento estocástico para los títulos nominales y reales, se denota por $M_{t}{ }^{n}$ y $M_{t}{ }^{r}$ respectivamente. El precio de un bono nominal que paga un peso en el tiempo $\tau$ y el precio de un bono real que paga una unidad de la canasta de consumo en el tiempo t deben satisfacer:

$$
P_{t}^{N}(\tau)=E_{t}^{P}\left[\frac{\mathrm{N}_{t+\tau}^{M}}{\mathrm{~N}_{t}^{M}}\right] P_{t}^{R}(\tau)=E_{t}^{P}\left[\frac{\mathrm{M}_{t+\tau}^{R}}{\mathrm{M}_{t}^{R}}\right]
$$

Por las estructuras de pago y asumiendo la no existencia de diferencias en los niveles de liquidez entre los bonos nominales y reales, el precio de la canasta de consumo que se denota como el conjunto de nivel de precios $Q_{t}$, es la razón entre el factor de descuento estocástico de los bonos reales y nominales:

$$
Q_{t}=\frac{M_{t}^{R}}{N_{t}^{R}}
$$

Según la dinámica que sigue el factor de descuento estocástico para los bonos reales y nominales, la solución que resuelve el sistema de ecuaciones diferenciales es el siguiente:

$d Q_{t}=\left(r_{t}^{N}-r_{t}^{R}\right) Q_{t} d t$

Así, después de integrar la expresión anterior y después de algunos cálculos algebraicos, el cambio marginal del nivel de precios futuros se expresa:

$\frac{Q_{t}}{Q_{t+\tau}}=e-\int_{t}^{t+\tau}\left(r_{s}^{N}-r_{s}^{R}\right) d s$

La conexión existente entre los rendimientos de los bonos reales y nominales en la curva cero-cupón y la esperanza de las expectativas de inflación puede expresarse en función de la descomposición del precio del bono nominal en la curva cero-cupón:

$P_{t}^{N}(\tau)=E^{t}\left[\frac{N_{N+\tau}^{M}}{N_{N}^{M}}\right]$
$=E^{P}\left[\frac{N_{N+\tau}^{M}}{N_{N}^{M}}\right] \times E^{P}\left[\frac{Q_{t}}{Q_{t+\tau}}\right] \times\left(1+\frac{\operatorname{cov}\left[\frac{N_{N+\tau}^{M}}{N_{N}^{M}}, \frac{Q_{t}}{Q_{t+\tau}}\right]}{E^{P}\left[\frac{N_{N+\tau}^{M}}{N_{N}^{M}}\right] \times E^{P}\left[\frac{Q_{t}}{Q_{t+\tau}}\right]}\right)$

Si se convierte este precio al rendimiento para un período de madurez $\mathrm{t}$, sacando el logaritmo natural a ambos lados de la expresión, se obtiene:

$y_{t}^{N}(\tau)=y_{t}^{R}(\tau)+\pi_{t}^{e}(\tau)+\phi(\tau)$

Donde la prima de riesgo por inflación es: $\phi_{t}(\tau)=-\frac{1}{\tau} \ln \left(1+\frac{\operatorname{cov}\left[\frac{M_{t+\tau}^{R}}{M_{t}^{R}}, \frac{Q_{t}}{Q_{t+\tau}}\right]}{E^{p}\left[\frac{M_{t+\tau}^{R}}{M_{t}^{R}}\right] \times E^{p}\left[\frac{Q_{t}}{Q_{t+\tau}}\right]}\right)$

La prima de riesgo por inflación depende del valor que pueda tomar $\operatorname{cov}\left[\frac{M_{t+\tau}^{R}}{M_{t}^{R}}, \frac{Q_{t}}{Q_{t+\tau}}\right]$, si esta expresión es negativa garantiza que la prima de riesgo inflacionaria tomará valores positivos durante la totalidad del período. La expresión es la covarianza entre el factor de descuento estocástico real y el nivel futuro de precios, de esto depende el grado de riesgo de los bonos nominales.

Finalmente, el BEl o la inflación compensada para un período de madurez $\tau$, queda definida como:

$B E I_{t}(\tau) \equiv y_{t}^{N}(\tau)-y_{t}^{R}(\tau)=\pi_{t}^{e}(\tau)+\phi_{t}(\tau)$

Definido como la diferencia entre los rendimientos nominales y reales para un mismo período de madurez descompuesto entre las expectativas promedio de inflación y la prima por riesgo inflacionario.

\section{Resultados y discusión}

En la estimación de los componentes de la curva de rendimientos (nivel, pendiente y curvatura) se hace uso del modelo estado espacio; para que el modelo empiece la recursión es necesario dar valores iniciales a los parámetros que se pretenden estimar, de tal forma en el presente documento se prueban una serie de valores iniciales ${ }^{6}$ consistentes teóricamente con los datos de mercado y con la teoría analizada, escogiendo aquellos en donde la medición de bondad de ajuste fue la mejor.

\subsection{Un análisis empírico del modelo dinámico libre de arbitraje de Nelson-Siegel (DNS), para los rendimientos nominales}

El vector de estado bajo un modelo DNS viene dado por los factores, NPC y es denotado como

$X_{t}^{N}=\left(N_{t}^{N}, P_{t}^{N}, C_{t}^{N}\right)$

Christensen, Diebold y Rudebusch (2007), demuestran que la tasa nominal libre de riesgo se toma como la suma de los factores nivel y pendiente:

$r_{t}^{N}=N_{t}^{N}+P_{t}^{N}$

Utilizando el filtro de Kalman, en la ecuación de estado

$X_{t}=(I-A) \mu+A X_{t-1}+n_{t}$

6. Se tomaron valores iniciales de los diferentes documentos en este trabajo, cuyo objetivo era el cálculo de las expectativas inflación descomponiendo la prima de riesgo por inflación, a partir de los datos en el mercado financiero. Melo y Granados (2010), Hordahl y Tristan, (2007), Christensen, Lópe y, Rudebusch (2010), Christensen, Diebold y Rudebusch (2010), Tobias y Hao Wu (2009), García y Werner (2010). 


\begin{tabular}{|c|c|c|c|c|c|c|c|c|}
\hline \multicolumn{4}{|c|}{ Matriz A } & \multirow{2}{*}{$\begin{array}{c}\text { Media } \\
\mu\end{array}$} & \multicolumn{4}{|c|}{ Matriz q } \\
\hline & $A .1\left(N_{t-1}\right)$ & A.2 $2\left(P_{t-1}\right)$ & A. $3\left(C_{t-1}\right)$ & & & $9.1\left(N_{t}\right)$ & $9.2\left(P_{t}\right)$ & $9 \cdot 3\left(C_{t}\right)$ \\
\hline \multirow[t]{2}{*}{ A1. $(N t)$} & 0.9798 & 0 & 0 & 0.1015 & q1. $\left(\mathrm{N}_{\mathrm{t}}\right)$ & 0.0316 & 0 & 0 \\
\hline & $(0.0141)$ & & & $(0.0050)$ & & $(0.0004)$ & & \\
\hline \multirow[t]{2}{*}{ A2. (Pt) } & 0 & 0.3924 & 0 & -0.0308 & q2. $\left(P_{t}\right)$ & 0 & 0.0190 & 0 \\
\hline & & $(0.0284)$ & & $(0.0023)$ & & & $(0.0004)$ & \\
\hline \multirow[t]{2}{*}{ A3. $(C t)$} & 0 & 0 & 0.0204 & -0.005 & q3. $\left(C_{t}\right)$ & 0 & 0 & 0.0168 \\
\hline & & & $(0.0426)$ & $(0.0036)$ & & & & $(0.0008)$ \\
\hline
\end{tabular}

* El panel del lado izquierdo consta de la matriz A y el vector de medias de los factores $\mu$. El panel del lado derecho contiene la matriz de choques entre los factores (Q). Los errores estándar de estos estimadores aparecen entre paréntesis debajo. El $\lambda \mathrm{N}$ estimado es 0.0609 .

Se estima el modelo usando el filtro de Kalman, que calcula estimadores consistentes y eficientes para los parámetros del modelo. El factor más persistente es el nivel y el menos persistente el de curvatura, como se observa en la matriz A, (la tasa de reversión media 0.9798 para el factor nivel y 0.0204 para el factor curvatura), la matriz de volatilidad $Q$, muestra que el factor que presenta una mayor exposición al choque es el nivel y el de menor es la curvatura, para las demás estimaciones. Se concluye que el promedio para el factor nivel es de $10.2 \%$, la media de la pendiente es de $-3.1 \%$ aproximadamente. Cabe anotar que el signo de la pendiente es negativo en el corto plazo, de modo que una pendiente promedio negativa implica que los rendimientos tienden a disminuir a medida que el período de madurez del título se extiende.

\subsection{Un análisis empírico del modelo dinámico libre de arbitraje de Nelson-Siegel (DNS) para los rendimientos reales}

Muy pocos documentos hacen referencia al comportamiento de los bonos reales por la escaza liquidez de estos (Shen 2006), por esta razón el rendimiento de los bonos reales se puede ver afectado. Debido a que los períodos de madurez para rangos muy altos en el mercado de los títulos reales es limitado, sólo tenemos en cuenta plazos de dos, tres y cinco años.

Utilizando el filtro de Kalman, en la ecuación de estado:

$$
X_{t}=(I-A) \mu+A X_{(t-1)}+n_{t}
$$

se obtienen las siguientes estimaciones para el modelo bajo títulos reales (Tabla 2).

La Tabla 2 muestra, que aunque el factor nivel tiene una mayor persistencia su valor no es tan alto (0.5396) como en los títulos nominales y la diferencia respecto al factor pendiente no es $\tan$ grande, la persistencia para el factor pendiente en títulos reales es de (0.3286), que es un valor muy parecido a la persistencia que presenta este factor en los bonos nominales. Ahora, en la matriz de variabilidad $q$, los dos factores evaluados tienen niveles de exposición al choque, similares ambos significativos. El factor nivel presenta un promedio de $5.1 \%$, un valor inferior al presentado para los títulos nominales; el factor pendiente presenta un promedio de $-3.3 \%$, que es prácticamente el mismo nivel promedio presentado por los títulos reales.

\begin{tabular}{|c|c|c|c|c|c|c|}
\hline & Matriz A & & Media & & Matriz q & \\
\hline & A.1(Nt-1) & A.2(Pt-1) & $\mu$ & & q.1 $(\mathrm{Nt})$ & $\mathrm{q} .2(\mathrm{Pt})$ \\
\hline A1. $\left(\mathrm{N}_{\mathrm{t}}\right)$ & 0.5396 & 0 & 0.0511 & q1. $\left(\mathrm{N}_{\mathrm{t}}\right)$ & 0.0267 & 0 \\
\hline & (0.0261) & & (0.0041) & & $(0.0004)$ & \\
\hline A2. $\left(P_{t}\right)$ & 0 & 0.3286 & -0.033 & q2. $\left(P_{t}\right)$ & 0 & 0.0189 \\
\hline & & (0.0288) & (0.0069) & & & $(0.0006)$ \\
\hline $\begin{array}{l}\text { * El pan } \\
\text { de medi } \\
\text { ne la m } \\
\text { estánda } \\
\text { debajo. }\end{array}$ & $\begin{array}{l}\text { el del lad } \\
\text { las de los } \\
\text { latriz de } \\
\text { El de est } \\
\text { El esti }\end{array}$ & $\begin{array}{l}\text { lo izquier } \\
\text { factores } \\
\text { choques } \\
\text { os estima } \\
\text { imado es }\end{array}$ & $\begin{array}{l}\text { do const } \\
\mu . \text { El pan } \\
\text { entre lo } \\
\text { adores } \\
0.0479 .\end{array}$ & $\begin{array}{l}\text { le la m } \\
\text { del lad } \\
\text { actores } \\
\text { recen }\end{array}$ & $\begin{array}{l}\text { atriz A y e } \\
\text { derecho } \\
\text { (q). Los } \\
\text { entre par }\end{array}$ & $\begin{array}{l}\text { I vector } \\
\text { contie- } \\
\text { errores } \\
\text { éntesis }\end{array}$ \\
\hline & & & Fuente: $R$ & OS, O.A. & y Girón, L. & E. (2013) \\
\hline
\end{tabular}

Gráficamente se confirma que el indicador BEI contiene de manera implícita, una prima de riesgo por inflación y que en general después de extraerla del BEI, la medida de las expectativas de inflación tiende a ajustarse mejor a la serie de inflación real [variación anual (\%)]. Este ajuste se observa de manera cercana para títulos con plazo de vencimiento a 5 años. (Gráfica 1)

En el rango de tiempo entre 2003 y 2007 las expectativas inflacionarias para la Gráfica 1 se ajustan bien a la inflación real; sin embargo, después de este período la inflación real crece muy por encima de las expectativas. No obstante se puede percibir el mismo patrón de comportamiento tendencial. (Gráfica 2)

En la Gráfica 2, se extrae de la serie de inflación real el comportamiento volátil de los alimentos. Se puede ver que las expectativas de los agentes tienden a ajustarse mejor descontado la prima de riesgo por inflación para el período de tiempo 2007 a 2010. 


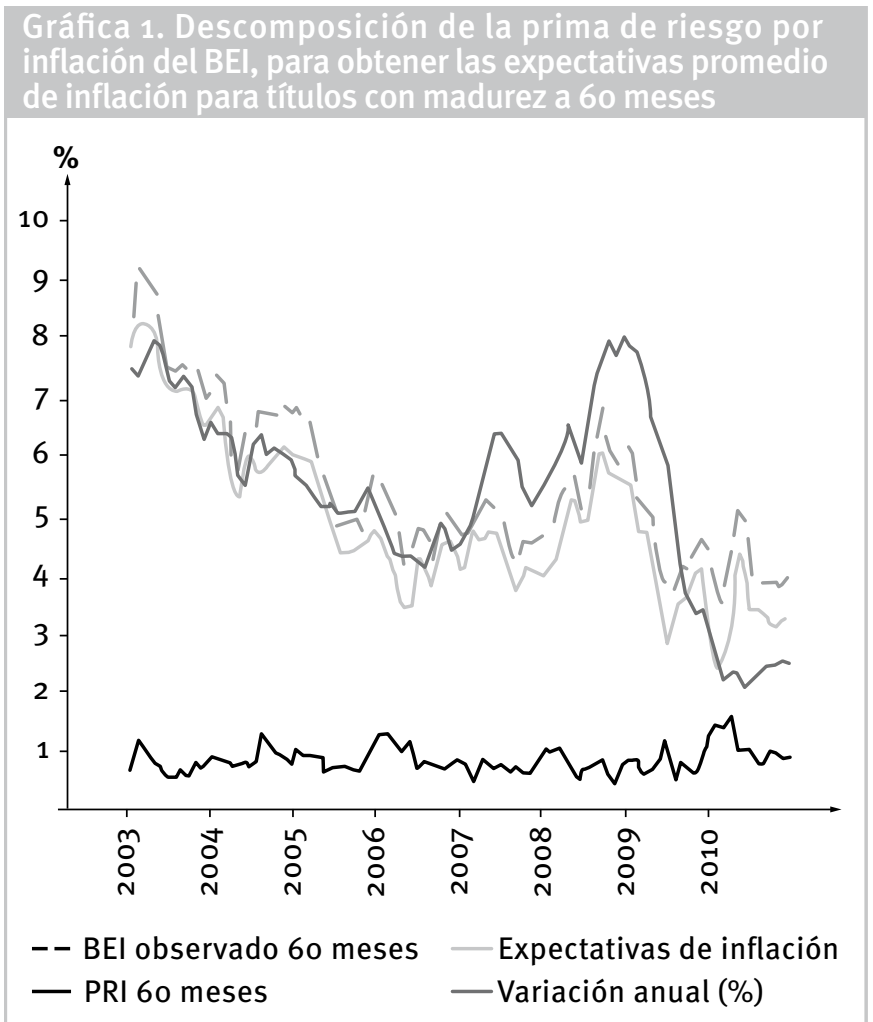

Fuente: Ríos, O.A. y Girón, L.E. (2013)

Gráfica 2. Descomposición de la prima de riesgo por inflación del BEI, para obtener las expectativas promedio de inflación para títulos con madurez a 60 meses (sin alimentos)

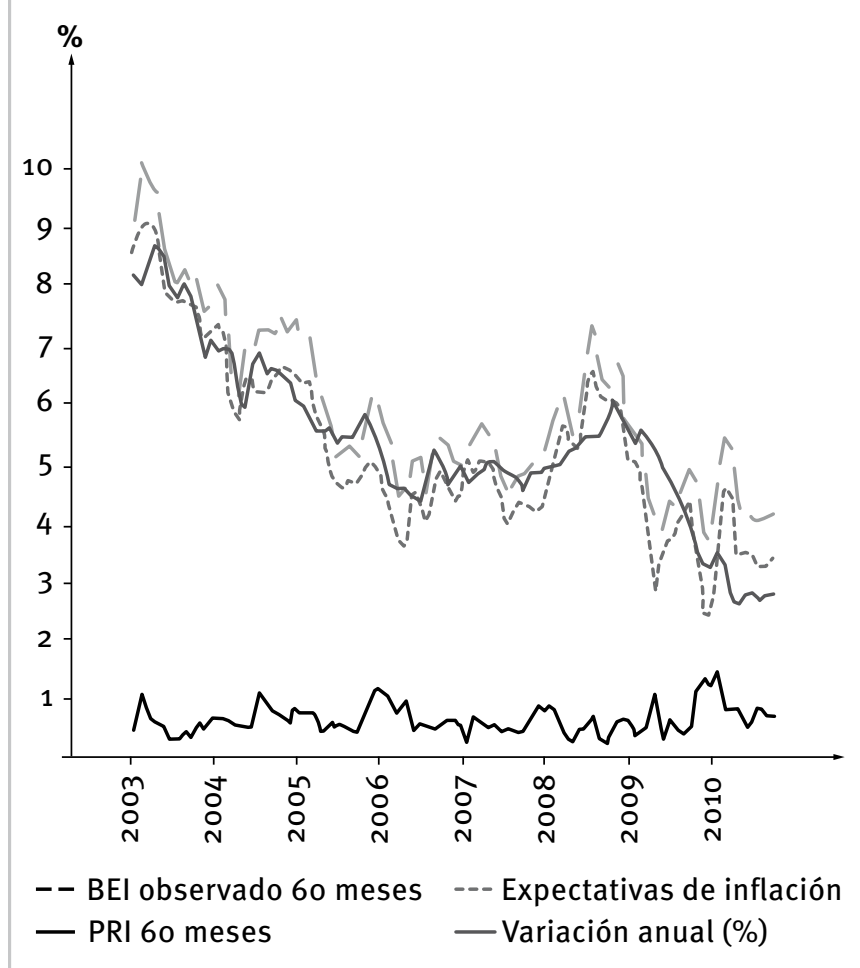

Fuente: Ríos, O.A. y Girón, L.E. (2013)
Gráfica 3. Comparación de las primas de riesgo por inflación para horizontes de 2, 3 y 5 años

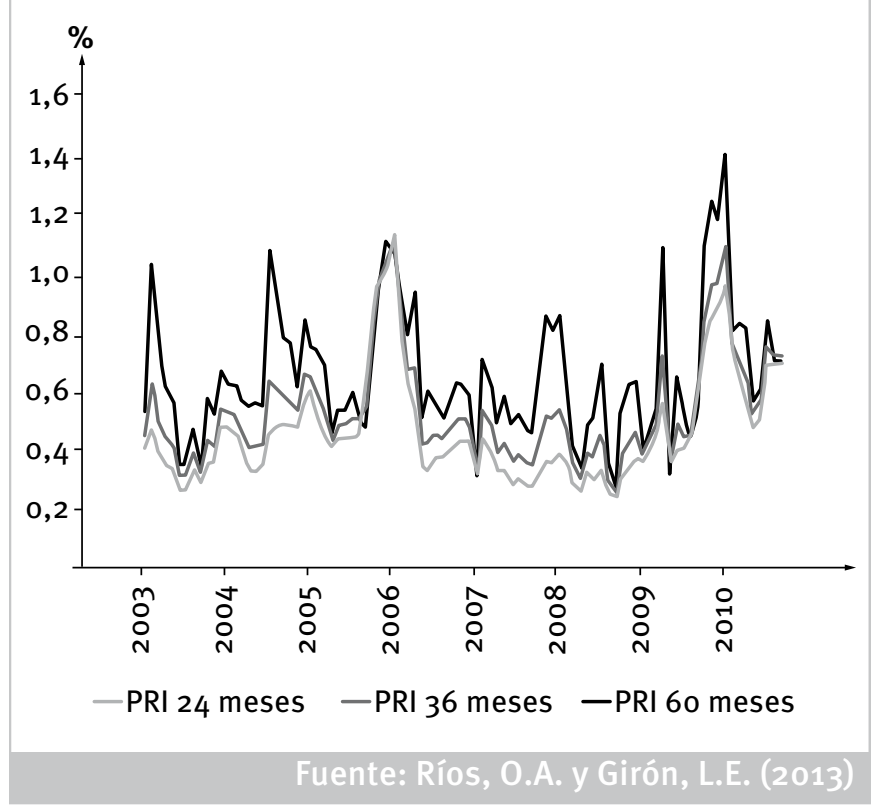

La Gráfica 3 presenta la comparación del comportamiento de las primas de riesgo por inflación a diferentes plazos. Se puede ver que para el mercado colombiano, la prima de riesgo ha presentado un comportamiento similar para los tres períodos de medición, además, consecuente con la teoría, a mayor período de vencimiento los agentes tienden a exigir una prima de riesgo por inflación más alta; también se puede observar que la prima de riesgo a 5 años, presenta picos más pronunciados, lo que podría entenderse a partir de su comportamiento que presenta una mayor variabilidad en el tiempo.

El resultado del promedio de la prima de riesgo por inflación es de 36.9455, 43,98506 y 53.18412 puntos básicos para 24,36 y 60 meses de plazo, se observa que el valor es mayor cuando aumenta el plazo de vencimiento del bono. Este resultado para la prima de riesgo inflacionario es lógico, debido a que el riesgo de cambios inesperados en la inflación se hace mayor conforme aumenta el período de madurez de un bono, por eso la prima pagada debe ser mayor.

Si se compara la estimación de la prima de riesgo por inflación (solo confrontable en los períodos de 24 y 60 meses), se puede observar que las mediciones presentes en Melo y Granados (2010), son un poco menores a las estimadas en este documento (alrededor de 1 punto básico), esta discrepancia se puede deber a que el número de parámetros necesarios para estimar las expectativas de inflación [objetivo real en el documento de Melo y Granados (2010)] es de un orden muy superior, además en este trabajo no se toman en cuenta las medidas de las encuestas de expectativas de inflación elaboradas por el Banco Central. Sin embargo, los resultados de este trabajo son consecuentes totalmente con el comportamiento del mercado sobre todo la prima de riesgo calculada para el largo plazo (5 años). 


\section{Conclusiones}

El resultado de la prima de riesgo por inflación obtenida con el modelo DNS, es consistente con los obtenidos con métodos más complejos; la diferencia en la estimación entre los métodos se encuentra alrededor de un punto básico. Desde el punto de vista práctico el inversionista logra una mejor comprensión del indicador con el presente método ya que todos los parámetros incluidos en su modelamiento corresponden a variables propias del mercado financiero.

Los resultados muestran que la prima de riesgo por inflación promedio para Colombia en los plazos de 2, 3 y 5 años es de 37, 44 y 53 puntos básicos, respectivamente, tomando como fuente estadística los rendimientos nominales y reales de los títulos emitidos por el Gobierno para los plazos descritos, los cuales en teoría representan cero riesgo.

Para el período entre 2003 y 2007 se puede evidenciar que las expectativas inflacionarias de los agentes se ajustan bastante bien al período en cuestión con la inflación observada; después de este período las expectativas no se ajustan de manera eficiente al comportamiento real del cambio en los precios. Para lograr un mejor ajuste para todo el período, es necesario aislar el componente volátil de alimentos, siendo el mayor plazo, 5 años, la de mejor ajuste.

El resultado de la prima de riesgo por inflación es mayor conforme crece el plazo de vencimiento, debido a que el riesgo de cambios inesperados en la inflación se hace mayor conforme aumenta el período de madurez debido a incrementos en la incertidumbre.

Este trabajo se desarrolló para Colombia en un período de relativa estabilidad inflacionaria; sin embargo el modelo utilizado también puede ser aplicado en futuras investigaciones en contextos de hiperinflación o devaluación en los cuales se esperaría que la prima de riesgo por inflación cambie dependiendo del escenario en la que se calcule.

\section{Referecias}

Ang, A., Bekaert, G. \& Wei, M. (2005). Do macro variables asset markets or surveys forecast inflation better? NBER Working Paper Series, 15. Washington, D.C., USA: Federal Reserve Board.

Adrian, T. \& Wu, H. (2009). The term structure of inflation expectations Report No 362. New York, USA: Federal Reserve Bank of New York.

Barro, J. (1976). Rational expectations and the role of monetary policy. Journal of Monetary Economics, 2 (pp. 1-32).

Christensen, H.E., Diebold, X. \& Rudebusch, G. (2010). The affine arbitrage-free class of nelson-siegel term structure models. San Francisco, USA: Federal Reserve Bank of San Francisco.

Christensen, H.E., López, J.A. \& Rudebusch, G. (2010). Inflation expectations and risk premiums in an arbitrage-free model of nominal and real bond yields. Paper 2007-20. San Francisco, USA: Federal Reserve Bank of San Francisco.

Deacon, M. \& Derry, A. (1994). Deriving estimates of inflation expectations from the prices of uk government bonds. Documento de trabajo. London, England: Bank of England.

Diebold, X., Rudebusch, G. \& Aruoba, S. (2005). The macroeconomy and the yield curve: a dynamic latent factor approach. Journal of Econometrics, 131 (pp. 309-338).

Fisher, I. (1930). The theory of interest. New York, USA: Macmillan.

García, J.A. \& Werner, T. (2010). Inflation risk and inflation risk premia Working Paper Series, 1162, Frankfurt, Germany, European Central Bank.

Gaviria, A. \& Téllez, S. (2010). Expectativas de Inflación en Colombia. Vniversitas económica, 10 (12) (pp. 1-17).

González, E., Jalil, M. y Romero, J.V. (2010). Inflación y expectativas de inflación en Colombia. Borradores de Economía (618). Bogotá, Colombia: Banco de la República.

Hordahl, P. \& Tristani, O. (2007). Inflation risk premia in termstructure of interestrates. Working paper series (734). Frankfurt, Germany: European Central Bank.

Litterman, R. \& Scheinkman, J.A. (1991). Common factors affecting bond returns. Journal of Fixed Income, (61) (pp. 54-61).

Lucas, R. (1972). Expectations and the Neutrality of Money. Journal of Economic Theory, (4) (pp. 103-124).

Lucas, R. (1976). Econometric policy evaluation: a critique. CarnegieRochester Conference Series on Public Policy, 1 (1) (pp. 19-46).

Melo, L.F. y Granados, J.C. (2010). Expectativas y prima por riesgo inflacionario bajo una medida de compensación a la inflación. Banco de la República, Borradores de Economía 589.

Misas, M. \& Vásquez, D.M. (2002). Expectativas de inflación en Colombia: un ejercicio econométrico. Banco de la República, Borradores de Economía 212.

Muth, F.J. (1961). Rational expectations and the theory of price movements. Econometrica, 29 (3).

Neumann, J. \& Morgenstern, O. (1944). Theory of games and economic behavior. New Jersey, USA: Princeton University Press.

Ramírez, F. (2007). Conceptos y construcción de la curva de rendimiento de TES en Colombia con las metodologías de Nelson-Siegel y Svensson. Medellin, Colombia: Universidad de Medellín.

Sack, B. (2000). Deriving inflation expectations from nominal and inflation-indexed treasury yields. Washington, USA: Board of Governors of the Federal Reserve.

Shen, P. (2006). Liquidity risk premia and breakeven inflation rates. Denver, USA: Federal Reserve Bank of Kansas City.

Shen, P. (2009). Developing a liquid market for inflation - indexed, Government Securities. Denver, USA: Federal Reserve Bank of Kansas City.

Soderlind, P. (2009). Inflation risk premia and survey evidence on macroeconomic uncertainty. San Francisco, USA: International Journal of Central Banking.

Tobias, A. \& Hao, W. (2009). The term structure of inflation expectations. Federal Reserve Bank of New York, Staff Report № 362.

Cuadernos de Administración / Facultad de Ciencias de la Administración / Universidad del Valle Periodicidad: semestral / ISSN impreso Nº120-4645- ISSN electrónico N²256-5078 / Nombre abreviado: cuad.adm. Edición Vol. 29 Nº 49 (enero - junio de 2013)

Prima de riesgo por inflación calculada con el Break-Even Inflation y el modelo dinámico Nelson-Siegel/Omar Alexander Ríos y Luis Eduardo Girón 